\title{
Table ronde sur « Mythe et histoire »
}

\section{Robert Ellrodt}

\section{(2) OpenEdition \\ Journals}

Édition électronique

URL : http://journals.openedition.org/shakespeare/508

DOI : 10.4000/shakespeare.508

ISSN : 2271-6424

Éditeur

Société Française Shakespeare

Édition imprimée

Date de publication : 1 novembre 1984

Pagination : 189

Référence électronique

Robert Ellrodt, «Table ronde sur « Mythe et histoire » », Actes des congrès de la Société française

Shakespeare [En ligne], 5 | 1984, mis en ligne le 01 janvier 2007, consulté le 06 mai 2019. URL : http:// journals.openedition.org/shakespeare/508; DOI : 10.4000/shakespeare.508 
SOCIETE FRANÇAISE SHAKESPEARE

Actes du Congrès 1983

\section{MYTHE ET HISTOIRE}

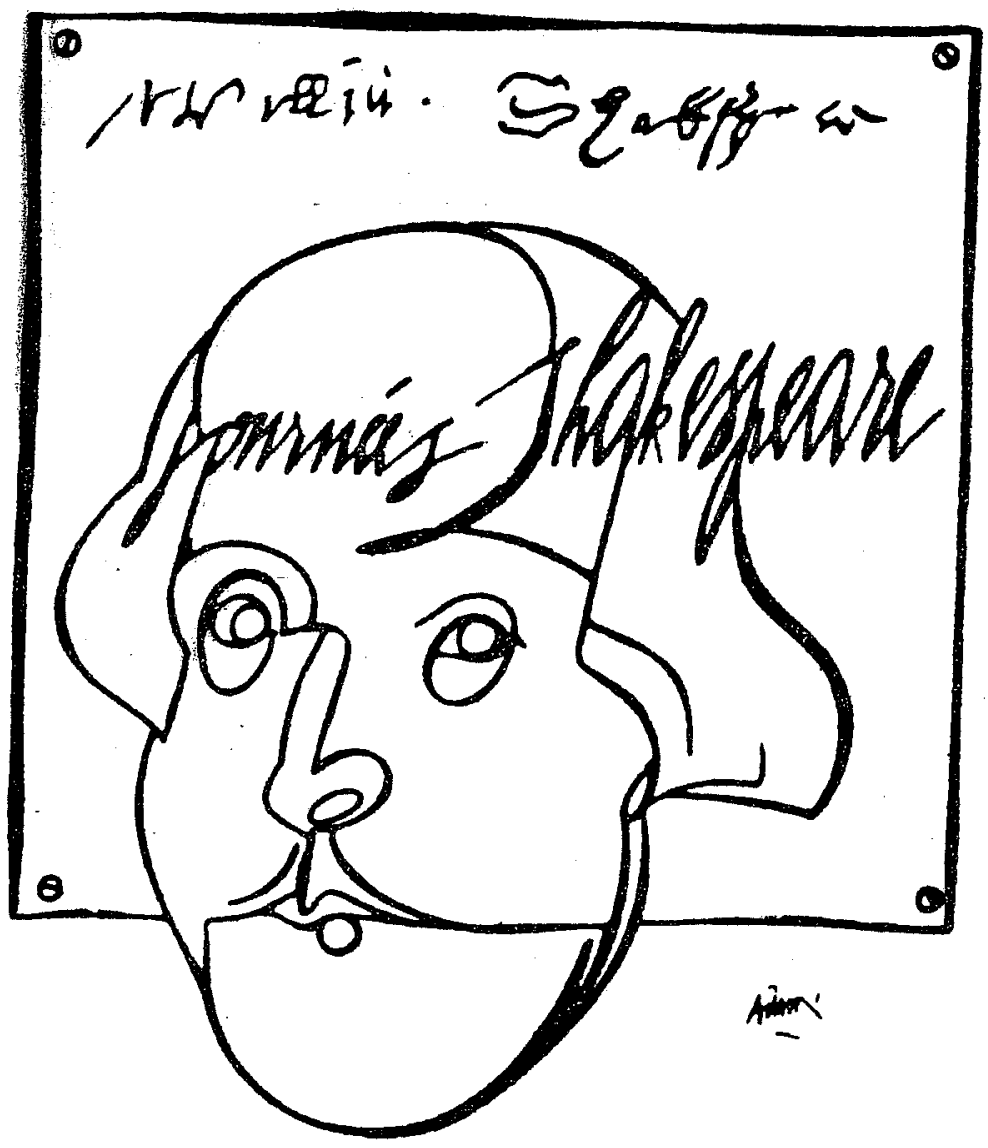

$$
\begin{array}{r}
\text { DiReCteur DE LA' PUblicatic } \\
\text { M.T. Jones-Davie }
\end{array}
$$

Publié avec le concours du Centre National de la Recherche Scientifiq

$$
\text { JEAN TOUZOT Libraire - Editeur }
$$

38, rue Saint-Sulpice 75278 PARIS CEDEX 06199 


\title{
TABLE RONDE SUR MYTHE ET HISTOIRE
}

\author{
Résumer ce débat animé se révèle impossible. Nous avons \\ demandé à chaque participant de fournir un condensé \\ de certaines de ses interventions.
}

\section{Robert ELLRODT}

Une distinction entre mythe cosmogonique, mythe politique et mythe poétique est utile. A la première catégorie appartiennent les mythes de renaissance, ainsi que le mythe du «royal scapegoat» quand le roi est divin. Le mythe chrétien s'y rattache. J'appelle mythe politique un mythe construit à des fins particulières. Les ethnologues ont découvert dans les sociétés africaines des mythes justificateurs de chaque nouveau pouvoir. L'origine ou la propagation de certains mythes étaient-elles différentes dans l'Angleterre de la Renaissance ? Quant au my the poétique il naît quand le poète parvient à créer un mythe original, indépendant ou non des mythes existants, cosmogoniques ou politiques. Sidney l'avait souligné : «a Tragedie is tied to the laws of Poesie, and not of Historie; not bound to follow the storie, but, having liberty, either to faine a quite newe matter, or to frame the history to the most tragicall conveniencie».

Qu'est-ce que l'histoire pour un homme du XVle siècle ? Shakespeare emploie le terme «history» au sens des «Histoires tragiques» de Bandello, mais le meurtre d'Edouard, événement historique, peut s'exprimer à travers le mythe d'Icare (3 H.VI, V.vi, 21-8). La vision providentielle de l'histoire (vision augustinienne pervertie par Eusèbe et Orose) sert à la fois à rendre l'histoire intelligible et à l'exploiter politiquement. Le recours à l'analogie ou à la typologie est spontané, parfois naïf : à la mort d'Elisabeth on s'émerveille que la Reine Vierge, née la veille de la fête célébrée pour la naissance de la Vierge, meure la veille 
de 1'Annonciation à Richmond, où son grand-père était né, etc. (Harrison, A Jacobean Journal, 28 avril 1603). $\mathrm{La}$ démythification de 1'histoire, très avancée en France et en Italie, est moins rapide en Angleterre où l'histoire moralisée est mise au service d'une cause par Buchanan comme par More. Rappeler ces faits et ce climat, ce n'est pas imposer une lecture «providentielle» ou «tyllardienne» de l'oeuvre shakespearienne, bien au contraire. C'est mettre en évidence la lucidité critique et la hardiesse du dramaturge dans des pièces historiques qui, sans renoncer à des analogies mythiques (par ex.«Jerusalem», 2 H.IV, IV. v, fin), éclairent d'un jour réaliste les motivations des personnages. Les «histories» peuvent se lire dans l'esprit de Bacon dénonçant dans l'histoire civile «les passions partisanes ou les créations de l'imagination» (sans oublier que Bacon lui-même n'hésitait pas pour flatter le roi à forger un «mythe jacobéen» dans son projet d'Histoire de la Grande Bretagne). Mais le moment shakespearien est un moment de transition, le plus propice à l'épanouissement de la tragédie s'il est vrai, comme l'écrit Vernant, que le tragique apparaît quand la distance est assez courte entre la tradition mythique et l'émergence de la pensée philosophique, politique et juridique.

Il n'est donc pas surprenant que succèdent aux «histories» ces tragédies, non moins historiques, où s'accomplit la transfiguration de l'histoire en mythe poétique. Mais si Cléopâtre mythifie Antoine dès l'acte IV (xv, 62-8), elle se déclare dans le même moment «No more but e'en a woman, and commanded / By such poor passion as the maid that milks . ..» (73-4), et la vision de la reine transfigurée dans la mort nous présente «a lass unparalleled》 (V. ii. 310). La réalité de l'histoire et la vérité du mythe s'unissent dans la poésie shakespearienne.

\section{Pierre SAHEL}

1) S'agissant des pièces historiques de Shakespeare, il est bon d'en découvrir les richesses propres. Chacune d'elles est une entité autonome, et point n'est besoin 
d'aller chercher ailleurs qu'en elle-même des éléments susceptibles de justifier son existence en tant qu'oeuvre d'art. Si des mythes y apparaissent, si une conception de l'histoire s'y fait jour, c'est que le drame les sécrète. Comme toujours dans Shakespeare, the play's the thing, et aucun postulat sur l'ingérence envahissante en son sein de l'idéologie dite dominante au temps de Shakespeare n'est recevable, pas plus que ne sont admissibles les prétentions critiques de l'élucider par une doctrine qui lui serait étrangère.

2) Toute généralisation sur le concept de mythe dans Shakespeare est difficile ou aléatoire. S'il est un aspect dont je décèle la récurrence, c'est bien l'ambivalence du mythe. Lors de ma communication sur les Henri $V I$, j'ai essayé de démontrer l'antagonisme, dans ces pièces, du mythe et de l'histoire ; j'ai cependant mentionné un trait tout différent de leur interaction : il arrive que l'histoire se serve du mythe, se l'allie et l'exploite. On peut constater la juxtaposition de deux idées contradictoires sur le mythe dans bien d'autres pièces shakespeariennes. Ainsi dans Antoine et Cléopâtre, l'image mythisée de la Cléopâtre plus que divine voguant sur le Cydnus coexiste avec la dramatisation de la jument d'âge avancé dont Antoine est loin d'avoir été l'unique étalon ; dans Troilus et Cressida, le mythe de la Belle Hélène ne détruit pas la fade impression produite par le personnage d'une Hélène à la beauté passée lors de sa seule apparition dans la pièce. L'exaltation voisine avec la subversion, le mythe avec le contremythe. Shakespeare façonne des mythes, en adapte et en intègre d'autres dans ses oeuvres, mais il se montre également critique devant la tendance mythogénique des hommes. Myth-maker, il est aussi un démythificateur de talent.

\section{Mme Dominique GOY-BLANQUET}

On trouve non pas un mais une quantité de «mythes Tudor» à l'époque où ont été composées les pièces historiques de Shakespeare. C'est une période où l'histoire se cherche, où les historiographes ne semblent pas tant 
préoccupés de justifier l'avènement de la dynastie Tudor que de trouver un système d'explication susceptible de réduire l'incohérence des faits qu'ils rapportent. Avec l'émergence d'une conscience historique nouvelle, ils s'occupent moins de disserter sur la prima causa et davantage d'analyser les causes secondes de l'histoire universelle, c'est-à-dire qu'au lieu d'invoquer à tout propos le mystère des plans divins, ils recherchent dans l'enchaînement des circonstances un processus logique naturel ; mais malgré leurs efforts de rationalisation, le providentialisme subsiste dans leurs écrits comme un système d'explication parallèle et concurrent. A la fin du XVIe siècle, même les plus scientifiques d'entre eux ne conçoivent guère une histoire humaine entièrement autonome, libre de toute intervention supérieure.

L'évolution de la conscience historique a été plus rapide en France, peutêtre, comme le suggère W. Smith Fussner, parce que le royaume était divisé en pays de droit écrit et pays de coutume, dualité qui encourageait les études comparatistes et la démarche critique; pour l'Angleterre, la loi commune à tout le royaume était inconstestablement d'origine divine, antérieure et supérieure à tout code écrit, qui ne pouvait être qu'humain. L'ouvrage de Sir Walter Ralegh, History of the World, constitue un catalogue exemplaire des croyances contemporaines et de leurs contradictions internes. Le grand mythe de la période, c'est cette conviction persistante que la Providence gouverne les affaires humaines.

Mais il est rare que ce mythe constitue un système d'explication cohérent, même chez les historiens qui croient à la transœndance, et ce n'est pas chez eux que Shakespeare a pu trouver un schéma déterministe ordonné : dans sa première tétralogie, la croissance régulière du mal jusqu'à sa résolution providentielle constitue un processus historique dont on chercherait vainement la trace dans les narrations dont il s'inspire; pour les chroniqueurs, le royaume se porte plus ou moins bien selon les jours, les talents individuels, et l'humeur de la Providence. Tandis qu'au théâtre, le mythe providentiel produit un schéma rigoureux: 
c'est le mythe qui fournit le cadre de référence, et qui opère la mise en ordre esthétique du désordre historique, sans qu'on puisse mesurer le degré d'adhésion requis.

\section{Jean FUZIER}

Henry $V$, pièce dont il n'a pas été question jusqu'ici au cours de colloque, est une oeuvre particulièrement intéressante au vu des rapports qui s'y établissent entre le my the et l'histoire. S'il est en effet un personnage historique de Shakespeare qui puisse revendiquer le statut de héros mythique, c'est bien le roi Henry $V$, dans la perspective épique d'une pièce dont on pourrait dire qu'elle est, à certains égards, l'lliade de Shakespeare. On peut remarquer cependant qu'à côté de la mythification sans réserves du personnage à laquelle contribue chacune des interventions du Choeur, qui constituent, avec certaines des tirades ou des actions prétées au héros, le discours mythifiant, il existe tout au long de l'oeuvre une sorte de contre-discours démythifiant qui humanise le personnage en nous révélant de sa part une certaine politique de non-responsabilité, un certain machiavélisme (même si c'est un machiavélisme du Bien) : il fait cautionner par l'Eglise la guerre qu'il va déclarer à la France et le bien-fon dé de cette guerre; il conduit habilement les traîtres Cambridge, Scroop et Gray à prononcer leur propre sentence; il se refuse, non sans quelque sophisme, à se sentir engagé par le sort des âmes des soldats qui périront au combat pour lui et pour l'Angleterre. Et ce ne sont là que les exemples les plus marquants. Que la figure mythique du personnage ne se trouve pas diminuée par cette humanisation, cela ne fait pas de doute (remarque de R. MARIENSTRAS), mais il convient de souligner que Shakespeare n'hésite pas à tempérer lé mythe par l'histoire, même si c'est à bâtir un my the qu'il travaille consciemment.

Jean-Marie MAGUIN

Avec Antony and Cleopatra, les pièces romaines 
offrent cet avantage de présenter l'instant où le mythe est créé, et les réactions que suscitent la proposition du mythe. C'est le moment où Cleopatra, s'adressant à Dolabella, lui décrit l'Antony de son rêve :

Cleo. You laugh when boys or women tell their dreams, Is't not your trick?

Dol I understand not, madam.

Cleo. I dreamt there was an Emperor Antony.

O such another sleep, that I might see

But such another man!

Dol. $\quad$ If it might please ye, - -

Cleo. His face was as the heavens, and therein stuck

A sun and moon, which kept their course, and

lighted

The little $\mathrm{O}$, the earth.

Dol. Most sovereign creature, -

Cleo. His legs bestrid the ocean, his rear'd arm

Crested the world: his voice was propertied

As all the tuned spheres, and that to friends :

But when he meant to quail, and shake the orb, He was as rattling thunder. For his bounty, There was no winter in 't: an autumn 'twas That grew the more by reaping: his delights Were dolphin-like, they show'd his back above The element they lived in: in his livery

Walk'd crowns and crownets: realms and islands

were

As plates dropp'd from his pocket.

Dol.

$$
\text { Cleopatra! }
$$

Cleo. Think you there was, or might be such a man As this I dreamt of ?

Dol. Gentle madam, no.

(V. 2. 74-94).

La réponse de Dolabella, «Gentle madam, no», est l'invariable réponse de l'Histoire-se-faisant à la proposi- 
tion du mythe. Et, bien entendu, l'habilité shakespearienne consiste à inscrire cette réponse plate dans une fin de pièce dont l'intrigue, parce que le personnage masculin principal est déjà mort, est propre, au contraire, à accréditer le mythe. Antoine, le soudard et le fêtard, n'est plus là pour décevoir ni Cléopatra ni le spectateur. Son apothéose peut donc avoir lieu et le mythe triompher de l'histoire. Les vers qui suivent (95-100), eux aussi très connus et souvent commentés, servent, dans la bouche de la reine d'Egypte, à condamner la vérité «naturelle» comme mensonge à l'égard des dieux et à regretter que la nature décevante ne se mêle pas de grandiose idéal pour se démarquer des ombres et chimères de l'imaginaire de deuxième classe.

\section{Henri SUHAMY}

Personne ne peut nier l'importance de la mythification dans les pièces historiques de Shakespeare. Mais il est temps de réagir contre une certaine tyrannie intellectuelle exercée par Tillyard et son école. Il est certes utile au spécialiste de connaitre tout ce qui concerne l'idéologie sur laquelle s'appuyait la monarchie des Tudors et des Stuarts, avec ses éléments mythiques, et de déceler leur présence dans l'oeuvre de Shakespeare. Il n'en est pas moins vrai que la partialité dogmatique de Tillyard aboutit à déformer la vérité, au moins par omission, sur deux points essentiels : elle ne tient pas compte de la contrepartie sceptique que Shakespeare ménage toujours, la dialectique de la crédulité et de l'incrédulité, la relativisation des croyances par le simple jeu dramatique. D'autre part on oublie quelquefois que les drames de Shakesperare sont d'abord des oeuvres d'art, et que méme s'ils ne sont pas coupés de toute signification, ni de la sensibilité nationale, les mythes qu'on y trouve ont essentiellement une fonction poétique, et non apologétique et extra-littéraire. 
Interventions sur deux points de détail

- A propos de la mythification d'Antoine, dans Antoine et Cléopâtre, on peut affirmer que celle-ci se produit dès le début de la pièce, ou même qu'elle constitue une donnée antérieure à la pièce. Le côté humain - trop humain - du personnage est confronté à son aspect quasidivin dès la première réplique.

- A propos d'Henry V, il semble que la mythification opérée par Shakespeare tend à l'idéalisation épique et royale du personnage, plutôt qu'à l'affirmation de sa légitimité dynastique. La prière du Roi :

Not to day, O Lord

O not to day, thinke not vpon the fault My Father made, in compassing the Crowne.

IV.i. 298

devait avoir pour effet de rappeler au spectateur l'illégitimité de la branche lancastrienne, non l'inverse.

\section{Richard MARIENSTRAS}

M. Robert Ellrodt a rappelé qu'au XVIe siècle, sur le continent et notamment en France, des historiens nombreux critiquaient les méthodes de l'histoire traditionnelle. Si l'idée d'une «histoire parfaite» n'avait pas germé en Angleterre, certains historiens y avaient attaqué des mythes historiques bien implantés, comme celui de la conquéte de l'Europe par le roi Arthur ou celui de l'origine troyenne de la monarchie anglaise. Ainsi, Polydore Vergil pratique la critique des sources même s'il ne dédaigne pas de faire oeuvre d'artiste et d'attribuer aux personnages historiques des discours inventés. Mais William Camden, déjà, refusera de le faire. Et il aura recours à des documents officiels pour écrire ses Annales du règne d'Elisabeth ${ }^{1}$.

L'histoire est donc en question au moment où Shakespeare écrit sa première tétralogie. Dans Richard $I I I^{2}$, un 
passage montre clairement qu'il lisait ses sources avec quelque recul et qu'il tenait à le faire savoir aux spectateurs :

[The young duke of ] York.

Marry, they say my uncle grew so fast

That he could gnaw a crust at two hours old :

'Twas full two years ere I could get a tooth.

Duchess. I prithee, pretty York, who told thee this?

York Grandam, his nurse.

Duchess. His nurse? Why she was dead ere thou wast born.

York. If 'twere not she, I cannot tell who told me.

(II, iv, 27-34)

C'est là une manière de faire douter de l'histoire par l'histoire, c'est-à-dire par une interrogation sur les sources, incorporée à la matière dramatique. Cette attitude critique, Shakespeare la prête également au jeune prince Edward qui, demandant si la tour de Londres fut bien construite par Jules César, précise ainsi sa question : «Is it upon record, or else reported/ Successively from age to age, he built it ?» (III, i, 72-73).

Pourtant, loin de conduire Shakespeare à mettre en doute ce qui peut-être méritait de l'être, ce regard critique lui permet au contraire de mieux accréditer non seulement les données fabuleuses ou historiques qu'il emprunte à ses sources, mais aussi des informations que ses sources ne lui fournissaient pas : ainsi, il rend Richard responsable du meurtre de Clarence. De même, dans Henry $V$, š̈l autorise, sur le mode mineur, quelques critiques démythifiantes contre le héros éponyme, il le glorifie sur le mode majeur et rejoint la tradition avec d'autant plus de force qu'il a laissé des doutes s'exprimer.

Richard III est un exemple frappant de création où l'art transforme en les magnifiant des matériaux qui passaient alors pour historiques. Shakespeare intègre le récit «véridique» de Hall dans une fresque plus stylisée dont 
la nécessité interne conduit à l'apparition du tyran parfait. La tétralogie est en effet construite sur une série de conflits qui opposent des adversaires de plus en proches par le sang : guerre entre nations d'abord, guerre civile entre familles cousines, guerre au sein de la méme famille, de la même faction, au coeur du personnage principal enfin. Richard est placé dans un schéma narratif qui fait converger sur lui tout le mal antérieurement réparti entre divers protagonistes. Le mariage incestueux que Richard projette rime avec ces conflits que la proximité des adversaires rend ignobles pour la nature. Et Shakespeare a puissamment utilisé ses difformités - la naissance monstrueuse, le bras trop court, la claudication, l'épaule trop haute - pour dessiner un monarque «inversé», définir enfin l'indéfinissable tyran contre lequel il est légitime de s'élever et de lutter, un tyran dont l'apparence, les attributs et l'action s'opposent presque point pour point à ceux du monarque légitime et suggèrent la silhouette de l'Antéchrist. Métamorphosant en mythe la fable et l'histoire, Shakespeare invente un espace historique où le dépassement de l'histoire par le mythe et l'enracinement du mythe dans l'histoire ne sont pas discernables - les vérités politiques, poétiques et historiques marchant pour ainsi dire d'un même pas, régi par quelque prodigieux principe d'immanence.

NOTES

1. Annales rerum Anglicarum et Hibernicarum regnante Elizabetha, Londres, 1615 (première partie); Leyden, 1625 (première et seconde partie. Il existe divverses traductions anglaises de cet ouvrage, ainsi qu'une traduction française publiée à Paris en 1627.

2. King Richard $I I I$, texte établi par Antony Hammond, The Arden Shakespeare, Methuen, Londres et New York, 1981. 